\title{
Electronic structure of liquid water from polarization-dependent two-photon absorption spectroscopy
}

\author{
Christopher G. Elles, Christopher A. Rivera, Yuyuan Zhang, Piotr A. Pieniazek, ${ }^{\text {a) }}$ and \\ Stephen E. Bradforth ${ }^{\text {b) }}$ \\ Department of Chemistry, University of Southern California, Los Angeles, California 90089, USA
}

(Received 29 November 2008; accepted 14 January 2009; published online 26 February 2009)

\begin{abstract}
Two-photon absorption (2PA) spectroscopy in the range from 7 to $10 \mathrm{eV}$ provides new insight on the electronic structure of liquid water. Continuous 2PA spectra are obtained via the pump-probe technique, using broadband probe pulses to record the absorption at many wavelengths simultaneously. A preresonance enhancement of the absolute 2PA cross section is observed when the pump-photon energy increases from 4.6 to $6.2 \mathrm{eV}$. The absorption cross section also depends on the relative polarization of the pump and probe photons. The variation of the polarization ratio across the spectrum reveals a detailed picture of the $2 \mathrm{PA}$ and indicates that at least four different transitions play a role below $10 \mathrm{eV}$. Theoretical polarization ratios for the isolated molecule illustrate the value of the experimental polarization measurement in deciphering the 2PA spectrum and provide the framework for a simple simulation of the liquid spectrum. A more comprehensive model goes beyond the isolated molecule picture and connects the 2PA spectrum with previous one-photon absorption, photoelectron, and x-ray absorption spectroscopy measurements of liquid water. Previously unresolved, overlapping transitions are assigned for the first time. Finally, the electronic character of the vertical excited states is related to the energy-dependent ionization mechanism of liquid water. (C) 2009 American Institute of Physics. [DOI: 10.1063/1.3078336]
\end{abstract}

\section{INTRODUCTION}

Two-photon excitation is a powerful spectroscopic tool for studying the electronic structure of a molecule because the selection rules are different for one- and two-photon transitions and because the relative polarization of the two photons often can be used to deduce the symmetry of the excited state. ${ }^{1-4}$ Although there have been numerous applications of two-photon excitation since the invention of the $Q$-switched laser, including considerable activity to develop two-photon dyes,${ }^{5}$ the continuous two-photon absorption (2PA) spectrum is rarely available. Recently, two schemes have been developed to record the continuous 2PA spectrum, one based on the Z-scan method using dispersed broadband laser pulses ${ }^{6-8}$ and the second derived from the broadband femtosecond pump-probe technique. ${ }^{9-12}$ Neither technique so far has been used to probe electronic transitions with one-photon energies in the vacuum UV, a region where many overlapping transitions often complicate the spectroscopy. In this paper we use 2PA spectroscopy based on intense ultraviolet (UV) pump pulses and multichannel continuum probing to reveal new details about the electronic structure of pure liquid water. Specifically, we report the continuous 2PA spectrum of water in the range from 7 to $10 \mathrm{eV}$ and use the polarization dependence of the spectrum to assign valence transitions in greater detail than was previously possible. Better knowledge of the electronic structure helps explain the unique ionization properties of liquid water, ${ }^{13-15}$ which has a vertical ionization

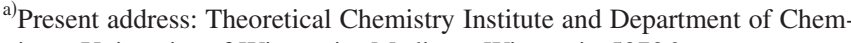
istry, University of Wisconsin, Madison, Wisconsin 53706.

b) Electronic mail: stephen.bradforth@usc.edu.
}

potential of $11 \mathrm{eV}$, but can be ionized adiabatically down to the optical absorption edge near $6-6.5 \mathrm{eV}^{16,17}$

One-photon absorption (1PA) spectroscopy of the isolated water molecule, for which hydrogen-bonding effects are absent, provides an important reference point for understanding the liquid spectrum. Figure 1(a) shows the gasphase absorption spectrum up to $10.5 \mathrm{eV}^{18}$ There are two broad absorption bands centered at 7.4 and $9.6 \mathrm{eV}$, and several sharp bands at higher energy. Each of the two lowest energy transitions, $3 s / 4 a_{1} \leftarrow 1 b_{1}\left(\widetilde{A} 1^{1} B_{1}\right)$ and $3 s / 4 a_{1} \leftarrow 3 a_{1}$ $\left(\widetilde{B} 2^{1} A_{1}\right)$, promotes an electron into the lowest unoccupied orbital of the water molecule. The $3 s / 4 a_{1}$ orbital can be described as having either antibonding valence character or dissociative $3 s$ Rydberg character. ${ }^{19-21}$ In contrast with the broad absorption bands for the two lowest excited states, both of which are repulsive along an $\mathrm{O}-\mathrm{H}$ bond, the sharp transitions at higher energy populate bound vibronic states with strong Rydberg character. Prominent among these are the $3 p_{z} / 5 a_{1} \leftarrow 1 b_{1}\left(2^{1} B_{1}\right)$ and $3 p_{x} / 2 b_{1} \leftarrow 1 b_{1}\left(3^{1} A_{1}\right)$ transitions at 10.0 and $10.2 \mathrm{eV}$, respectively. ${ }^{22}$ Figure 2 shows a few of the calculated molecular orbitals of gas-phase water and illustrates the five lowest energy transitions. Although dipole forbidden in the linear absorption spectrum, the twophoton allowed $3 p_{y} / 2 b_{2} \leftarrow 1 b_{1}\left(1^{1} A_{2}\right)$ transition is included in the figure. The $2 b_{2}$ orbital has both valence antibonding and $3 p_{y}$ Rydberg character. ${ }^{20,21}$

The linear absorption spectrum of liquid water [Fig. 1(b)] is very different from the gas-phase spectrum because of strong intermolecular interactions in the condensed phase. ${ }^{23-25}$ The $\tilde{A} 1^{1} B_{1}$ transition is the only clearly resolved absorption band and is $\sim 0.9 \mathrm{eV}$ higher in energy than in the 


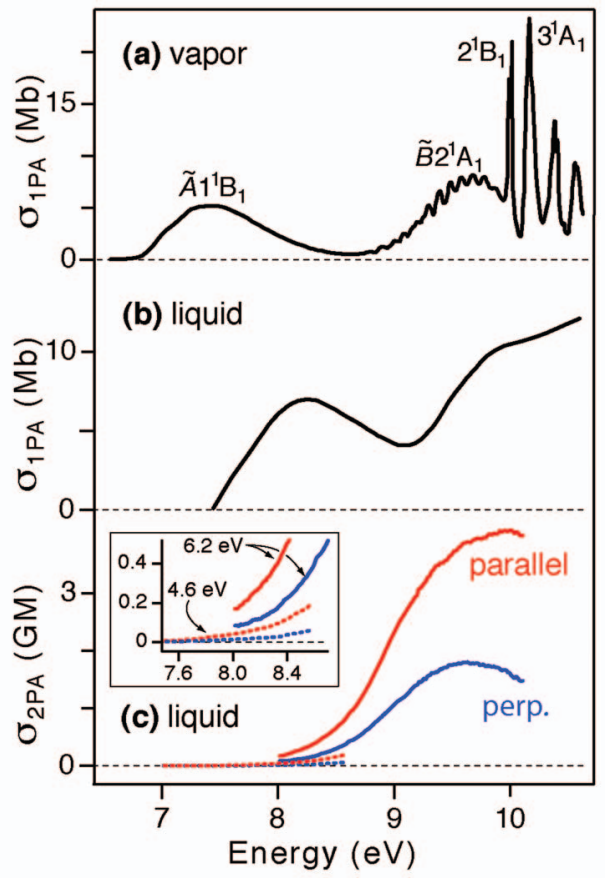

FIG. 1. (Color) 1PA spectrum of (a) gas-phase and (b) liquid-phase water from Refs. 18 and 24, respectively, and (c) 2PA spectrum of liquid water from this work. The two-photon spectrum is obtained for both parallel (red) and perpendicular (blue) relative polarization of the photons, and for pumpphoton energies of $4.6 \mathrm{eV}$ (dotted lines) and $6.2 \mathrm{eV}$ (solid lines). The inset shows the overlapping region of the 4.6 and $6.2 \mathrm{eV}$ spectra in greater detail.

isolated molecule. A broad shoulder near $9.8 \mathrm{eV}$ has been assigned as the $\widetilde{B} 2^{1} A_{1}$ transition, ${ }^{25-28}$ whereas there are no sharp resonances and the absorption intensity increases monotonically above $10 \mathrm{eV}$. Although the effect of solvation on Rydberg states is not known for certain, it has been suggested that compression of low-lying Rydberg orbitals by the solvent may broaden the transitions and shift them to higher energy. ${ }^{29,30}$ Complicating the picture for higher energy Rydberg states, however, recent photoelectron (PE) measurements show that the first ionization potential of water decreases from $12.6 \mathrm{eV}$ in the gas phase to only $11.2 \mathrm{eV}$ in the liquid. $^{31}$ An alternate view is one in which the excited molecular orbitals are partially delocalized over one or more neighboring solvent molecules. ${ }^{32-36}$ Unfortunately, a clear and consistent theoretical description of the electronically excited states of liquid water is not yet available because accurate electronic structure calculations are extremely difficult for such strongly interacting molecules. ${ }^{34-41}$

2PA spectroscopy provides a new window on the electronic transitions of liquid water because the symmetry selection rules are different than for linear spectroscopy. Absolute 2PA cross sections have been reported at a limited number of discrete energies. ${ }^{42-46}$ Most notably, Nikogosyan et $a l^{42}$ observed a monotonic rise of the cross section for degenerate two-photon excitation with a picosecond laser at five energies ranging from 7.8 to $9.3 \mathrm{eV}$. The increasing 2PA across that range strongly contrasts with the distinct absorption band at $8.3 \mathrm{eV}$ in the 1PA spectrum. Thomsen et al. ${ }^{28}$ obtained a more complete spectrum, although on a relative absorption scale, by using the pump-probe method to record the perpendicularly polarized $2 \mathrm{PA}$ on a point-by-point basis

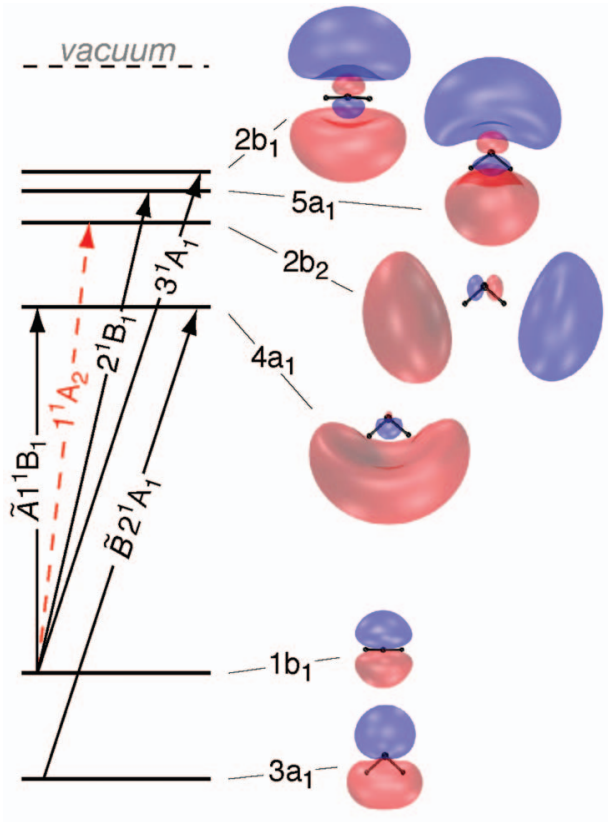

FIG. 2. (Color) The five lowest energy transitions and associated molecular orbitals for the isolated water molecule. Each transition is labeled according to the identity of the excited state. The $1^{1} A_{2}$ transition is one-photon forbidden.

from 7.9 to $10.7 \mathrm{eV}$. That work was the first to suggest that the 2PA reaches a maximum, or at least has a shoulder, near $9.8 \mathrm{eV}$. However, there is too much uncertainty between points in the spectrum to draw a clear conclusion, and there may be a relatively large background contribution from the photoproducts produced by two-photon ionization with the intense pump pulse. In this article we report, in the absence of photoproducts, the first 2PA spectrum of liquid water obtained at all wavelengths simultaneously.

Importantly, the 2PA transition strength depends on the polarization of the two photons being absorbed. The simultaneous absorption of two photons occurs through coupling of two transition dipole moments, thus the variation of the absorption cross section with the relative polarization of the two photons depends on the symmetry of the initial and final states. ${ }^{1,4}$ Monson and McClain ${ }^{1-3}$ elegantly demonstrated the polarization dependence of the cross section for an orientationally averaged sample and show that the polarization ratio reveals the symmetry of the electronically excited state in favorable cases. The polarization ratio is defined as $\sigma_{\text {para }} / \sigma_{\text {perp }}$, where $\sigma_{\text {para }}$ and $\sigma_{\text {perp }}$ are the 2PA cross sections for parallel and perpendicular relative polarization of the pump and probe photons. We show for water that a varying polarization ratio over the range from 7.5 to $10 \mathrm{eV}$ indicates that at least four different transitions contribute to the 2PA spectrum. The variation of the experimental polarization ratio allows us to separate those contributions and tentatively assign the transitions based on molecular symmetry arguments. Calculated 2PA cross sections for the gas-phase water molecule guide our assignment of the liquid spectrum. We also connect our results with the 1PA spectrum and with recent measurements of the X-ray absorption (XA) and PE spectra of liquid water, and discuss the electronic structure of water in the context of the ionization process. 


\section{METHODS}

\section{A. Experimental methods}

The 2PA spectrum of liquid water is measured by temporally and spatially overlapping two pulsed laser beams in an $\sim 75 \mu \mathrm{m}$ thick film of distilled water flowing between two tungsten wires. ${ }^{47}$ Electronic excitation occurs by simultaneous absorption of one deep UV pump photon and one probe photon, where attenuation of the broad-bandwidth probe beam reveals the $2 \mathrm{PA}$ cross section as a function of total excitation energy. ${ }^{10,11}$ Neither photon is absorbed alone.

Focusing a portion of the fundamental $800 \mathrm{~nm}$ light from a $1 \mathrm{kHz}$ regeneratively amplified Ti:sapphire laser (SpectraPhysics, Hurricane) into a $\mathrm{CaF}_{2}$ substrate generates supercontinuum probe light that spans the range from 1.8 to $3.9 \mathrm{eV}(690-315 \mathrm{~nm})$. A pair of aluminum-coated off-axis parabolic mirrors collimates and then focuses the probe to a spot size of $75 \mu \mathrm{m}$ at the sample. After the sample the broadband probe is dispersed with a diffraction grating and the entire spectrum is recorded simultaneously with $\sim 1.25 \mathrm{~nm}$ resolution on a 256 channel diode array. The third and fourth harmonic of the laser provide deep-UV pump photons. The $800 \mathrm{~nm}$ fundamental is doubled, then sumfrequency mixed with residual $800 \mathrm{~nm}$ to produce the third harmonic at $4.65 \mathrm{eV}(267 \mathrm{~nm})$. The third harmonic is used directly as pump light or sum frequency mixed with the remaining $800 \mathrm{~nm}$ to produce the fourth harmonic at $6.2 \mathrm{eV}$ $(200 \mathrm{~nm})$. We adjust the spot size and pulse energy of the pump beam in order to eliminate transient absorption contributions from the products of two-(pump)-photon ionization of water. ${ }^{13-15,28}$ The spot size of the pump beam is at least twice that of the probe, and the pump pulse energy is between 0.8 and $6.6 \mu \mathrm{J}$. The 2PA spectrum is obtained for parallel and perpendicular polarization by rotating the linear polarization axis of the $800 \mathrm{~nm}$ beam prior to continuum generation. The polarization purity is better than 200:1 across the probe spectrum, and is better than 70:1 for the $4.6 \mathrm{eV}$ pump, as measured by extinction of the light through a calcite polarizer. The purity of the $6.2 \mathrm{eV}$ pump light is at least 40:1, measured using a calibrated stack of nine silica plates at Brewster angle.

A two-dimensional representation of the time-dependent absorption signal for parallel polarization is shown in Fig. 3 . The group delay of the probe light arriving at the sample depends on the wavelength and leads to a curved spectrum in the figure. The line labeled " $\tau=0$ " indicates maximum temporal overlap of the pump and probe pulses as a function of wavelength. We integrate over the delay time to eliminate cross-phase modulation of the supercontinuum probe caused by nonresonant interaction with the intense pump laser pulse. $^{48}$ The sinusoidal cross-phase modulation, which is weak in the present example, vanishes entirely when we integrate over the time-dependent signal at each wavelength, leaving only the contribution from 2PA. The integration limits (dashed lines in Fig. 3) encompass the entire 2PA band, and, by varying those limits, we verify that transient photoproducts do not contribute to the integrated signal. Because the ionization yield is very large, the absence of solvated electrons due to two-photon ionization by the pump pulse

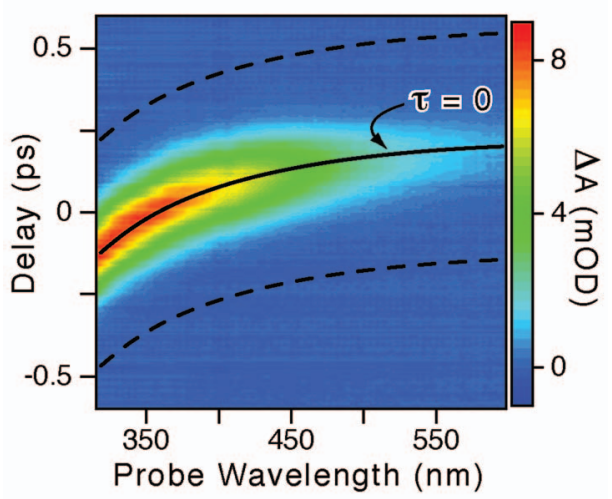

FIG. 3. (Color) Two-dimensional plot of the $2 \mathrm{PA}$ signal for $6.2 \mathrm{eV}$ pump and broadband probe. The solid line $(\tau=0)$ is the optimum temporal overlap of the pump and probe pulse at each wavelength; dashed lines are an example of the limits of integration used to obtain the 2PA cross section from Eq. (1) of the text.

ensures that the excited state population also is negligible. The extinction coefficient is almost certainly much smaller for the excited state than it is for the strongly absorbing solvated electron, and therefore excited state absorption does not contribute to the integrated 2PA signal when there is no electron signal.

The integrated absorption signal at each wavelength is proportional to the commonly used 2PA coefficient $\beta$,

$$
\beta=\frac{\ln (10)}{l f E_{\text {pump }}} \int \Delta A_{2 \mathrm{PA}}(\tau) d \tau,
$$

where $\Delta A_{2 \mathrm{PA}}(\tau)$ is the time-dependent absorption, $\tau$ is the delay time between pump and probe pulses, $l$ is the path length of the sample, $E_{\text {pump }}$ is the energy of the pump pulse in $\mu \mathrm{J}$, and $f$ is an overlap factor (with units $\mathrm{cm}^{-2}$ ) that accounts for the different diameters of the pump and probe beams. An equivalent result is obtained from the value of the 2PA signal at maximum overlap of the pump and probe pulses (i.e., $\tau=0$ ),

$$
\beta=\frac{\ln (10)}{l f E_{\mathrm{pump}}} \sqrt{2 \pi} w \Delta A_{2 \mathrm{PA}}(0) .
$$

Here, $w=\mathrm{FWHM} / \sqrt{8 \ln (2)}$ (FWHM denotes full width at half maximum) is the width of the pump-probe crosscorrelation peak at each wavelength. We prefer to use the integrated signal in order to eliminate possible contributions from cross-phase modulation and because it gives a better result for the very weak absorption on the low energy side of the 2PA spectrum. By integrating the signal we also avoid having to measure the cross-correlation width $w$ at each probe wavelength.

The 2PA coefficient is proportional to the absolute 2PA cross section $\sigma_{2 \mathrm{PA}}{ }^{12}$

$$
\sigma_{2 \mathrm{PA}}=\frac{\hbar \omega_{\text {pump }}}{n} \beta,
$$

where $\omega_{\text {pump }}$ is the frequency of the pump pulse and $n$ is the number density of water. The absolute cross section often is expressed in units of Goeppert-Mayer (1 GM $=10^{-50} \mathrm{~cm}^{4} \mathrm{~s}$ molecule ${ }^{-1}$ photon $\left.^{-1}\right)$. The most significant 
TABLE I. Calculated optical transition properties for water [only parallel 2PA cross sections are shown; polarization ratios $\left(\sigma_{\text {para }} / \sigma_{\text {perp }}\right)$ are in parentheses].

\begin{tabular}{|c|c|c|c|c|c|}
\hline & $\tilde{A} 1^{1} B_{1}$ & $1^{1} A_{2}$ & $\widetilde{B} 2^{1} A_{1}$ & $2^{1} B_{1}$ & $3^{1} A_{1}$ \\
\hline \multicolumn{6}{|c|}{ Isolated water } \\
\hline Transition energy & $7.61 \mathrm{eV}$ & $9.36 \mathrm{eV}$ & $9.88 \mathrm{eV}$ & $10.01 \mathrm{eV}$ & $10.23 \mathrm{eV}$ \\
\hline 1PA oscillator strength, $f$ & 0.051 & $\ldots$ & 0.058 & 0.008 & 0.043 \\
\hline \multicolumn{6}{|l|}{$2 \mathrm{PA}$ cross section, ${ }^{\mathrm{a}} \sigma_{\text {para }}$} \\
\hline Degenerate pump $/ 10^{-3} \mathrm{GM}$ (polarization ratio) & $2.4(1.33)$ & $30.8(1.33)$ & $40.5(2.28)$ & $33.4(1.33)$ & $182.8(8.75)$ \\
\hline $4.6 \mathrm{eV}$ pump $/ 10^{-3} \mathrm{GM}$ (polarization ratio) & $2.5(1.26)$ & $30.8(1.33)$ & $41.5(2.28)$ & $34.9(1.30)$ & $192.3(8.48)$ \\
\hline $6.2 \mathrm{eV}$ pump $/ 10^{-3} \mathrm{GM}$ (polarization ratio) & $4.5(0.84)$ & $52.0(1.01)$ & $58.1(2.33)$ & 49.3(1.09) & 233.1(7.55) \\
\hline \multicolumn{6}{|c|}{ Liquid water ${ }^{\mathrm{b}}$} \\
\hline Transition energy & $8.09 \mathrm{eV}$ & $9.74 \mathrm{eV}$ & $10.01 \mathrm{eV}$ & $10.53 \mathrm{eV}^{\mathrm{c}}$ & $10.25 \mathrm{eV}^{\mathrm{c}}$ \\
\hline \multicolumn{6}{|l|}{$2 \mathrm{PA}$ cross section, ${ }^{\mathrm{a}} \sigma_{\text {para }}$} \\
\hline Degenerate pump/10-3 GM & $4.0 \pm 0.1$ & $43.1 \pm 0.6$ & $64 \pm 1$ & $35 \pm 1$ & $110 \pm 2$ \\
\hline
\end{tabular}

2PA cross-sectional units are Goeppert-Mayer $\left(1 \mathrm{GM}=10^{-50} \mathrm{~cm}^{4} \mathrm{~s}\right.$ molecule ${ }^{-1}$ photon $\left.^{-1}\right)$.

${ }^{\mathrm{b}}$ Liquid-phase cross sections are taken from Ref. 39 and converted from atomic units to GM as described in that work.

${ }^{c}$ We identify the states at 10.25 and $10.53 \mathrm{eV}$ as $3^{1} A_{1}$ and $2^{1} B_{1}$, respectively, by comparing their relative cross sections with the gas-phase values. The states were not explicitly assigned in Ref. 39.

source of uncertainty in our absolute cross-sectional measurements is the spatial overlap of the pump and probe beams at the sample. Measuring the horizontal and vertical beam diameters at the sample, by using the knife-edge technique and assuming Gaussian beam profiles, we account for the relative spot sizes of the pump and probe via the factor $f$ in Eq. (1). The probe beam diameter sometimes varies slightly with wavelength even though it is focused with an achromatic parabolic mirror; therefore several independent measurements of the spectrum are necessary in order to confirm that the shape of the spectrum is not distorted by poor pump-probe overlap. Expanding the pump beam diameter as much as possible mitigates this effect. We estimate that the uncertainty of our absolute cross-sectional measurements is $\pm 20 \%$, although the relative error across the spectrum is much smaller. The polarization ratio measurements described below do not depend on knowing the absolute cross section and are the result of many back-to-back measurements of the relative parallel and perpendicular absorption spectra.

\section{B. Computational methods}

Rotationally averaged 2PA cross sections for the five lowest electronically excited states of the isolated water molecule are calculated using the DALTON electronic structure package. ${ }^{49}$ The calculations use coupled cluster with single and double substitutions (CCSD) response theory with the $d$-aug-cc-pVTZ basis set, identical to that of Thomsen et $a l .{ }^{28}$ We calculate parallel and perpendicular cross sections at eight different pump-photon energies in addition to the degenerate pump-probe energy for each transition. The sum of pump and probe photon energies is always equal to the transition energy (i.e., there is no broadening of the spectral lines). All calculations are performed at the experimental equilibrium geometry of the isolated water molecule, with $\mathrm{O}-\mathrm{H}$ bond lengths equal to $0.9585 \AA$ and a bond angle of $104.34^{\circ} .50$

\section{RESULTS}

\section{A. Calculated two-photon cross sections}

Gas phase calculations highlight several important aspects of 2PA spectroscopy and are a helpful reference for understanding the experimental 2PA spectrum of liquid water. Table I shows the calculated 2PA cross sections for each of the five lowest transitions of the isolated water molecule. We obtain nearly the same results as Thomsen et al. ${ }^{28}$ for degenerate excitation, in which both pump and probe photons have equal energy, except that we use a slightly different geometry for our calculations. Our calculations additionally include nondegenerate excitation in which the pump-photon energy is fixed and the probe energy is adjusted for each of the five states individually in order to give the total transition energy. As the energy of either photon approaches onephoton resonance with the lowest excited state $(7.6 \mathrm{eV}$ for the isolated molecule), the 2PA transition strength increases due to preresonant enhancement. Similar to the more familiar case of preresonant enhancement of Raman cross sections, the increase in 2PA strength comes from the resonance term in the denominator of the expression for the two-photon transition dipole tensor elements,

$$
M_{f \leftarrow g}^{\alpha, \beta}=\sum_{k}\left(\frac{\left\langle g\left|\mu_{\alpha}\right| k\right\rangle\left\langle k\left|\mu_{\beta}\right| f\right\rangle}{\omega_{k g}-\omega_{\text {pump }}+i \Gamma}+\frac{\left\langle g\left|\mu_{\beta}\right| k\right\rangle\left\langle k\left|\mu_{\alpha}\right| f\right\rangle}{\omega_{k g}-\omega_{\text {probe }}+i \Gamma}\right) .
$$

The indices $\alpha$ and $\beta$ refer to the polarization axes of pump and probe photons with frequency $\omega_{\text {pump }}$ and $\omega_{\text {probe }}$, respectively. $\omega_{k g}$ is the transition frequency between ground state $g$ and intermediate state $k, 1 / \Gamma$ is proportional to the lifetime of $k$, and $f$ refers to the final state. Our calculations do not include the factor $\Gamma$, it is shown here only to illustrate that the tensor elements remain finite in the limit of one-photon resonance.

The cross section of the $\widetilde{A} 1^{1} B_{1}$ transition increases by nearly a factor of 2 when changing from 4.6 to $6.2 \mathrm{eV}$ pump photons because of the resonance term (see Table I). The 


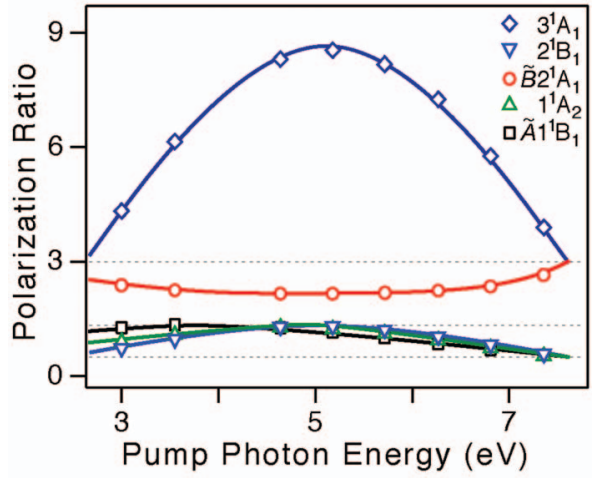

FIG. 4. (Color) Calculated energy dependence of the 2PA polarization ratio $\left(\sigma_{\text {para }} / \sigma_{\text {perp }}\right)$ for each of the five lowest energy transitions of the isolated water molecule. Solid lines are to guide the eye and thin dashed lines represent limiting values of the ratio for degenerate and near-resonant excitation.

increase is less dramatic for the highest energy $3^{1} A_{1}$ transition because the $5.6 \mathrm{eV}$ probe photon is already nearer the resonance condition at the lower pump-photon energy, in which case the second term in Eq. (4) dominates the expression. The 2PA cross section of all five states increases as the energy of the pump (or probe) photon approaches resonance with the lowest excited state.

The calculated cross sections $\sigma_{\text {para }}$ in Table I are for parallel polarization of the pump and probe beams. Using the cross sections for perpendicular polarization (not shown), we calculate the ratio $\sigma_{\text {para }} / \sigma_{\text {perp }}$ of each transition. Values for this polarization ratio are given in parentheses in the table. As the pump energy increases from 4.6 to $6.2 \mathrm{eV}$ the polarization ratio decreases for all of the calculated transitions, except for the $\widetilde{B} 2^{1} A_{1}$ transition, where the ratio increases slightly. Figure 4 shows the behavior of the polarization ratio of each state over a larger range of photon energies. Even without referring to the $a b$ initio calculations, a few of the points in the figure can be assigned a priori based on the $C_{2 v}$ symmetry group of the water molecule. ${ }^{1}$ For example, in the limiting case of one-photon resonance (i.e., a pump energy of $7.6 \mathrm{eV}$ ) the polarization ratio is equal to $\frac{1}{2}$ for the relevant nontotally symmetric ( $A_{2}$ or $B_{1}$ symmetry) two-photon transitions and it is equal to 3 for the totally symmetric $\left(A_{1}\right.$ symmetry) two-photon transitions. Another special case is degenerate excitation, in which case symmetry arguments require that the polarization ratio is equal to $4 / 3$ for all nontotally symmetric transitions, but totally symmetric transitions can have any value greater than $4 / 3$.

The polarization ratios in Fig. 4 demonstrate the limiting behavior in the cases of degenerate and resonant excitation. For nontotally symmetric transitions the ratio varies from a maximum value of $4 / 3$ at the point of degenerate excitation to a minimum value of $\frac{1}{2}$ when either the pump or probe photon is resonant with a one-photon-allowed transition. On the other hand, totally symmetric transitions have a polarization ratio that is always larger than $4 / 3$, and is equal to 3 in the one-photon resonance limit. Interestingly, the symmetric $\widetilde{B} 2^{1} A_{1}$ and $3^{1} A_{1}$ transitions have very different behavior in the intermediate range. The ratio for the $\widetilde{B} 2^{1} A_{1}$ transition decreases to a value of 2.28 , but the ratio for the $3^{1} A_{1}$ tran- sition increases to 8.75 . The large polarization ratio for the $3^{1} A_{1}$ transition may be a consequence of the strong $p_{x}-p_{x}$ atomic character of the transition. Within a purely atomic limit the structure of the two-photon transition dipole tensor ${ }^{2}$ is such that the two-photon $p_{x}-p_{x}$ transition vanishes for perpendicularly polarized photons, and the polarization ratio $\left(\sigma_{\text {para }} / \sigma_{\text {perp }}\right)$ becomes infinitely large.

Condensed phase calculations from Paterson et al. ${ }^{39}$ are included in Table I for comparison with the gas-phase values. Those authors obtain transition energies and two-photon cross sections from CCSD response theory using a hybrid quantum-classical approach that treats a single water molecule as a quantum mechanical solute (with the $d$-aug-cc-pVDZ basis set) in a bath of classical water molecules. The condensed phase calculations reveal a shift to slightly higher energy for all five transitions. The 2PA intensities also change slightly from the gas to the liquid. Cross sections were not reported for perpendicular polarization, and therefore it is not clear how the liquid environment affects the calculated polarization ratio.

\section{B. Experimental two-photon absorption spectrum and polarization ratio}

The experimental 2PA spectrum of liquid water is presented in Fig. 1(c), where the absolute cross section is plotted as a function of the total two-photon energy for both parallel and perpendicular polarization. The absorption is stronger for parallel than for perpendicular polarization across the entire spectrum. Spectra are obtained using two pump-photon energies, 4.6 and $6.2 \mathrm{eV}$. The spectrum generated with the lower energy pump photons spans the range from 7.0 to $8.6 \mathrm{eV}$, whereas the higher photon energy gives a spectrum covering 8.0-10.2 eV. Reflecting preresonant enhancement, the cross section in the overlapping region is different for the two pump-photon energies [see Fig. 1(c), inset]. The cross section is approximately three to five times larger for the parallel spectrum and four to seven times larger for the perpendicular spectrum when they are obtained with the higher energy pump photons. The absolute cross sections that we measure are about an order of magnitude larger than the values from the gas-phase calculation and slightly larger than those of Nikogosyan et al., ${ }^{42,51}$ but they are within the range of values measured more recently with femtosecond laser pulses at $8.5,{ }^{44} 8.8,{ }^{43} 9.3,{ }^{45}$ and $9.4 \mathrm{eV}^{46}$

The monotonic rise of the absorption up to $9.5 \mathrm{eV}$ is qualitatively consistent with previous experimental results ${ }^{28,42}$ and theoretical calculations, ${ }^{39}$ but the actual shape of our perpendicularly polarized spectrum is slightly different from the one obtained by Thomsen et al. ${ }^{28}$ Their spectrum rises more rapidly from 8.5 to $9.5 \mathrm{eV}$, and peaks at a slightly higher energy. We believe their spectrum may be slightly distorted due to wavelength-dependent overlap of the pump and probe beams and a possible contribution from transient photoproducts. ${ }^{52}$ The former is a likely consequence of measuring the spectrum on a point-by-point basis, whereas the latter is a result of the high intensity pump pulses that those authors use to intentionally generate and study the products from two-(pump)-photon ionization of the 


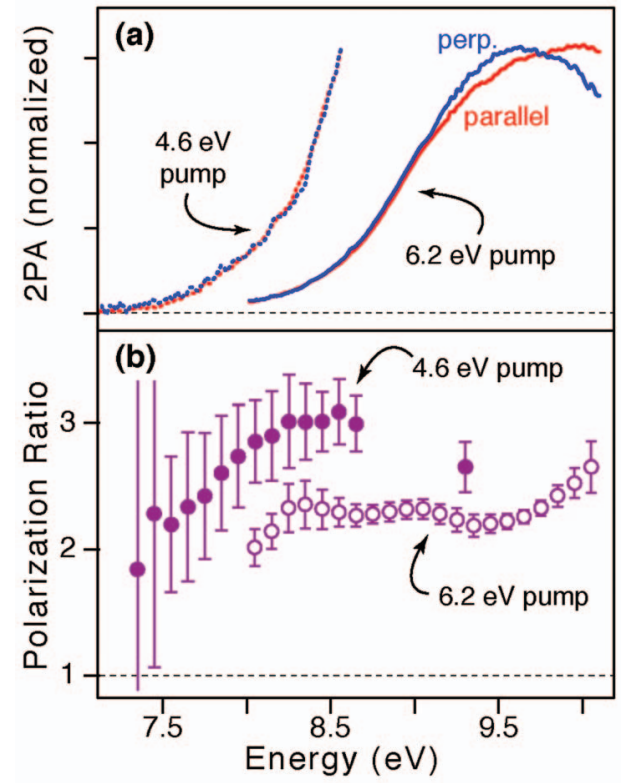

FIG. 5. (Color) Normalized 2PA spectra (a) and polarization ratios (b). The parallel (red) and perpendicular (blue) spectra are obtained for pump-photon energies of $4.6 \mathrm{eV}$ (dotted lines; closed circles) and $6.2 \mathrm{eV}$ (solid lines; open circles).

liquid. In our experiment, we benefit from collecting the entire 2PA spectrum simultaneously and from carefully avoiding photoproducts and excited state absorption by using low intensity pump pulses.

Figure 1(c) shows that the shape of the 2PA spectrum depends on the relative polarization of the pump and probe beams. Most notably, the perpendicular spectrum peaks near $9.6 \mathrm{eV}$, whereas the parallel spectrum reaches a maximum near $9.8 \mathrm{eV}$. The shift would be difficult to discern without recording the continuous $2 \mathrm{PA}$ spectra. To highlight the difference, Fig. 5 shows the normalized spectra and corresponding polarization ratios $\sigma_{\text {para }} / \sigma_{\text {perp. }}$. In addition to the different peak positions, there is a subtle but reproducible difference below $8.3 \mathrm{eV}$, where the perpendicular spectrum does not decay to the baseline as quickly as the parallel spectrum. The different behaviors of the spectra below $8.3 \mathrm{eV}$ are most clearly evident from the varying polarization ratio in Fig. 5(b).

The polarization ratio helps visualize differences between the parallel and perpendicular spectra. For the $4.6 \mathrm{eV}$ pump energy, the ratio increases from a value of roughly 2 to a value of $3.0( \pm 0.3)$ because of the different rising edge behaviors of the two spectra. The polarization ratio obtained using the higher energy pump photons also increases slightly from 8.1 to $8.3 \mathrm{eV}$, then has a relatively constant value of 2.3 $( \pm 0.1)$ in the range from 8.3 to $9.0 \mathrm{eV}$, decreases gently in the region around $9.4 \mathrm{eV}$, and increases again above that energy. The varying ratio above $9 \mathrm{eV}$ mirrors the different peak positions in Fig. 5(a). The ratios in the figure are an average of many independent measurements, with error bars representing one standard deviation from the mean. The lower spectral resolution compared to the 2PA spectra is a result of deliberately averaging over several adjacent pixels when taking the ratio of the parallel and perpendicular spectra.
The polarization ratio was also recorded at discrete energies of 9.3 and $10.8 \mathrm{eV}$ using pump-photon energies of 4.6 $\mathrm{eV}(267 \mathrm{~nm})$ and $6.2 \mathrm{eV}(200 \mathrm{~nm})$, respectively, and a $4.6 \mathrm{eV}$ probe pulse. The value of the first measurement, $2.7( \pm 0.2)$, is included in the figure, but the other, $2.4( \pm 0.2)$, is not shown because it is at much higher energy. A striking result from Fig. 5(b) is the different ratios obtained for 4.6 and $6.2 \mathrm{eV}$ pump photons in the regions where the data overlap. ${ }^{53}$ Resonance enhancement reduces the polarization ratio for the latter, which is the same result that we obtain for four of the five transitions from our gas-phase calculation in Table I.

\section{ANALYSIS AND DISCUSSION}

\section{A. Two-photon absorption spectrum}

The spectra in Fig. 1 highlight an important difference between one- and two-photon excitations of liquid water. The strong 1PA band near $8.3 \mathrm{eV}$ is conspicuously absent from both the parallel and perpendicular 2PA spectra. The absence of a strong absorption band from the latter two is consistent with the gas-phase calculations in Table I. Unlike one-photon excitation, the calculated two-photon cross section is an order of magnitude weaker for the $3 s / 4 a_{1} \leftarrow 1 b_{1}$ $\left(\widetilde{A} 1^{1} B_{1}\right)$ transition than it is for higher energy transitions. In the Rydberg picture of the isolated molecule, both the upper and lower orbitals of the transition have most or all of their amplitude on the oxygen atom, and the weak $2 \mathrm{PA}$ is due to the atomic $s \leftarrow p$ character of the $\tilde{A} 1^{1} B_{1}$ excitation. (The nonbonding $1 b_{1}$ molecular orbital is essentially oxygen $2 p_{x}$.) The transition would be strictly two-photon forbidden if the atomic-level selection rule was valid, but all two-photon transitions are rigorously symmetry allowed within the $C_{2 v}$ point group. Nevertheless, a weak $4 a_{1} \leftarrow 1 b_{1}\left(\widetilde{A} 1^{1} B_{1}\right)$ transition in the liquid suggests that the atomic character of the transition is not significantly altered.

Analyzing the spectrum of liquid water at higher energies is more complicated because of the relative lack of structure and the larger number of states that potentially play a role. Thomsen et al. ${ }^{28}$ suggested that the 2PA shoulder or peak near $9.8 \mathrm{eV}$ is related to the shoulder in the same region of the 1PA spectrum. Those authors also infer from their experimental polarization ratio of $2.7( \pm 0.5)$ that a single transition to the $\widetilde{B} 2^{1} A_{1}$ state dominates the $2 \mathrm{PA}$. Calculated polarization ratios for the lowest five transitions in the isolated water molecule provided the justification of their assignment because only the $4 a_{1} \leftarrow 3 a_{1}\left(\widetilde{B} 2^{1} A_{1}\right)$ transition has a comparable ratio. In contrast, our sensitive broadband measurements reveal that a single transition is not adequate to reproduce the 2PA spectrum. For example, even though the perpendicular 2PA spectrum peaks near $9.6 \mathrm{eV}$, the parallel 2PA spectrum reaches a plateau or maximum near $9.8 \mathrm{eV}$. The discrepancy indicates that multiple transitions contribute to the spectrum in this energy range, and highlights how little is known about the character of the excited states for oneand 2PA of liquid water. ${ }^{13-15}$ 


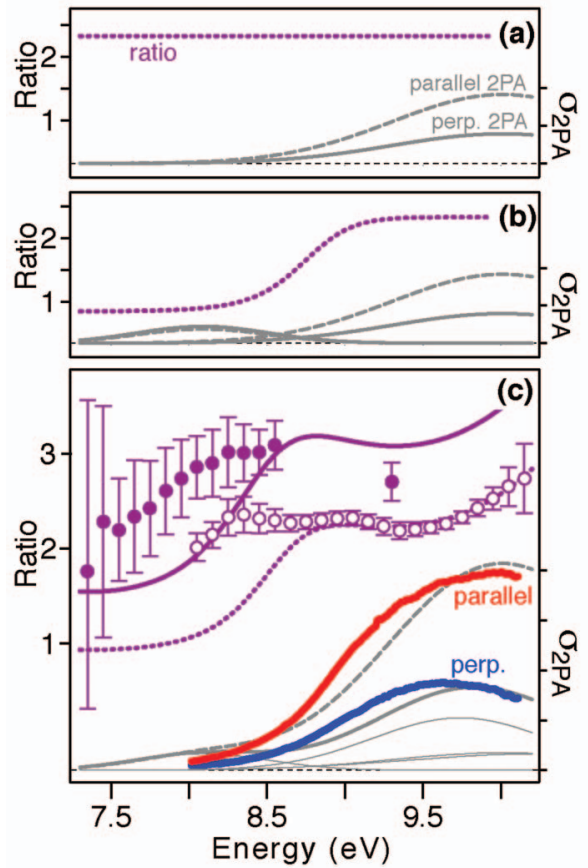

FIG. 6. (Color) Simulation of the polarization ratio (dotted purple line) for 2PA of water using 1, 2, or 4 states (a, b, and c, respectively). Thick gray lines in each panel are for parallel (dashed line) and perpendicular (solid line) polarization, with each of the four individual components of the perpendicular 2PA spectrum shown in the bottom panel (thin gray lines). The bottom panel compares the simple simulation results with the experimental data, where only the spectra obtained with a pump-photon energy of $6.2 \mathrm{eV}$ are shown.

\section{B. Simulation of the 2PA spectrum and polarization ratio}

\section{Simple model of polarized two-photon absorption}

The following examples show that the spectrally resolved polarization ratio measurement is a sensitive tool for analyzing the 2PA spectrum when there are overlapping transitions. A key point is that at least four transitions are necessary to reconstruct the general features of the polarization ratio for liquid water. For example, a single transition would give a constant ratio across the entire spectrum. We illustrate this point in Fig. 6(a) using the $\widetilde{B} 2^{1} A_{1}$ transition with an arbitrary (Gaussian) bandwidth of $1.6 \mathrm{eV}$. The ratio is solely determined by the relative 2PA cross sections for parallel and perpendicular polarization. Instead, the energy-dependent ratio changes smoothly from one value to another after adding a contribution from a second state, such as $\widetilde{A} 1^{1} B_{1}$, which has a slightly stronger absorption in the perpendicular spectrum than in the parallel spectrum [Fig. 6(b)]. The rate of change and the position of the rising edge depend on the two contributing states and their relative intensities. Using calculated polarization ratios for the gas-phase transitions, for example, the lower energy transition in the figure has a polarization ratio of only 0.84 but the overall ratio increases to a value of 2.33 in the region where $\widetilde{B} 2^{1} A_{1}$ dominates the spectrum.

We observe a qualitatively similar behavior of the experimental polarization ratio below $8.5 \mathrm{eV}$, where the ratio increases from less than 2 to about 3 when obtained with a pump-photon energy of $4.6 \mathrm{eV}$. The changing polarization ratio indicates that at least two states contribute to the $2 \mathrm{PA}$ spectrum of water in this region. Although the two transitions in Fig. 6(b) qualitatively reproduce the rise of the experimental polarization dependence, those two transitions alone cannot explain the energy dependence above $9 \mathrm{eV}$ or the different peak positions of the parallel and perpendicular 2PA spectra. At least two additional transitions are required to reproduce all features of the experimental polarization ratio, including not only the dip near $9.4 \mathrm{eV}$ and the increasing ratio above that energy, but also the relatively flat ratio from 8.3 to $9 \mathrm{eV}$. Thus, we simulate the polarization ratio using a total of four transitions.

We start with a simple model of the 2PA spectrum of liquid water that uses the four lowest energy transitions from the liquid-phase calculations of Paterson et al. ${ }^{39}$ That work considers the perturbation of the gas-phase transitions in going to the liquid at the level of a single quantum mechanical water molecule surrounded by a classical solvent. Paterson et al. did not explicitly identify the transitions with respect to their gas-phase analogs, but we identify the fourth transition at $10.25 \mathrm{eV}$ as correlating to the $3^{1} A_{1}$ state based on the relatively large $2 \mathrm{PA}$ cross section that is similar to the gasphase result for that state (see Table I). The three lowest transitions are in the same order for both environments. To simulate the polarization ratio we use the calculated values from the isolated molecule because perpendicular 2PA cross sections were not reported for the liquid. We assume that each transition has a Gaussian line-shape with bandwidth $\geq 1 \mathrm{eV}$ in the liquid. ${ }^{54}$

Figure 6(c) compares our experimental results with the simulated polarization ratios and 2PA spectra from this simple model of a "central" solvated water molecule. The only way to reproduce the experimental dip at $9.4 \mathrm{eV}$ using the calculated transition energies from Paterson et al. ${ }^{39}$ and our gas-phase polarization ratios is to allow the low energy tail of one or both of the $A_{1}$-symmetry transitions to extend below the lower energy, nontotally symmetric $1^{1} A_{2}$ transition. Therefore, we use a bandwidth that gradually increases from 1 to $2 \mathrm{eV}$ when going from $\tilde{A} 1^{1} B_{1}$ to the highest energy transition $\left(3^{1} A_{1}\right)$. Within this simple model, the dip in the ratio is a result of $1{ }^{1} A_{2}$ having a narrower bandwidth, as well as a lower polarization ratio, compared to the totally symmetric $\widetilde{B} 2^{1} A_{1}$ and $3{ }^{1} A_{1}$ transitions at higher energy. The simulation successfully reproduces the larger polarization ratio for $4.6 \mathrm{eV}$ compared to $6.2 \mathrm{eV}$ pump photons. A decreasing ratio with increasing pump-photon energy is the calculated result for all of the transitions except $\widetilde{B} 2^{1} A_{1}$ (see Fig. 4), indicating once again that $\widetilde{B} 2^{1} A_{1}$ most likely is not the dominant transition in the experimental 2PA spectrum.

Two major shortcomings of this simple simulation of the polarization ratio are a rising edge at too high of an energy and failure to reproduce the flat ratio in the range from 8.3 to $9 \mathrm{eV}$. More importantly, the simulated 2PA spectra in Fig. 6(c) do not match the individual experimental absorption spectra. Adding a contribution from the fifth state in the calculations cannot solve the problem. Clearly a simple simulation using the cross sections and polarization ratios from the isolated molecule is not sufficient to describe the 
electronic structure of liquid water. This is merely a perturbed gas-phase model for water, in which the transition energies and bandwidths are modified according to the approximate picture of a single central water molecule being solvated in the liquid.

\section{Comprehensive spectroscopic model}

A more refined approach to simulating the experimental 2PA spectrum is one that is guided by other spectroscopic measurements of liquid water, rather than relying entirely on calculations. Specifically, the transition energy and bandwidth of the $\tilde{A} 1^{1} B_{1}$ state are known from the 1PA spectrum ( 8.3 and $1.0 \mathrm{eV}$, respectively), and we can place the $\widetilde{B} 2{ }^{1} A_{1}$ transition energy using the photoelectron (PE) spectrum of liquid water. PE spectroscopy gives the energies of the occupied molecular orbitals of liquid water with respect to vacuum. ${ }^{31,55}$ Because both the $\widetilde{A} 1^{1} B_{1}$ and $\widetilde{B} 2{ }^{1} A_{1}$ states have the same $3 s / 4 a_{1}$ upper orbital, the PE spectrum reveals their relative optical transition energies by showing the relative location of the $1 b_{1}$ and $3 a_{1}$ lower orbitals. ${ }^{15}$ In fact, electronic coupling of water molecules in the condensed phase causes the collection of $3 a_{1}$ orbitals to split into two components, $3 a_{1}$ and $3 a_{1}{ }^{*}$, representing bonding and antibonding combinations. ${ }^{55,56}$ The splitting presumably results in two optical transitions, $4 a_{1} \leftarrow 3 a_{1}{ }^{*}$ and $4 a_{1} \leftarrow 3 a_{1}$. Using the relative energies of $1 b_{1}, 3 a_{1}{ }^{*}$, and $3 a_{1}$ from the PE spectrum, as well as the energy of the $4 a_{1} \leftarrow 1 b_{1}\left(\widetilde{A} 1^{1} B_{1}\right)$ transition in the 1PA spectrum, we predict optical transition energies of about 9.8 and $11.4 \mathrm{eV}$ for the two components of the $4 a_{1}$ $\leftarrow 3 a_{1}^{*} / 3 a_{1}\left(\widetilde{B} 2^{1} A_{1}\right)$ transition. The lower transition energy of $9.8 \mathrm{eV}$ is consistent with previous assignments of the shoulder in the 1PA spectrum as the $\widetilde{B} 2{ }^{1} A_{1}$ state, ${ }^{25-28}$ although to our knowledge this is the first time such an assignment has been justified using the PE spectrum. The higher energy $4 a_{1} \leftarrow 3 a_{1}$ component is outside of the range of our experiment.

Those values for the $\widetilde{A} 1{ }^{1} B_{1}$ and $\widetilde{B} 2{ }^{1} A_{1}$ transitions are the starting point for a second simulation of the 2PA spectrum. Once again, we use a total of only four states in the simulation because that is the minimum number of transitions required to reproduce the general features of the experimental polarization ratio. We vary the remaining parameters, including the transition energies and intensities of the two additional states, in order to reproduce the experimental results in Fig. 7. The parameters for the simulation are in Table II. The upper component of the $\widetilde{B} 2^{1} A_{1}$ transition (at $11.4 \mathrm{eV}$ ) is well outside of the range of our experiment and does not play a role in the simulated spectrum. Inspired by the decreasing polarization ratio with increasing pump-photon energy in our calculations, we assume that resonance enhancement has a larger impact on perpendicular than parallel transitions. An additional enhancement of the perpendicular transitions by a factor of 1.3 more than the parallel transitions is consistent with the absolute cross sections in the overlapping region of the experimental spectrum [see Fig. 1(c), inset] and reproduces the reduced polarization ratio for

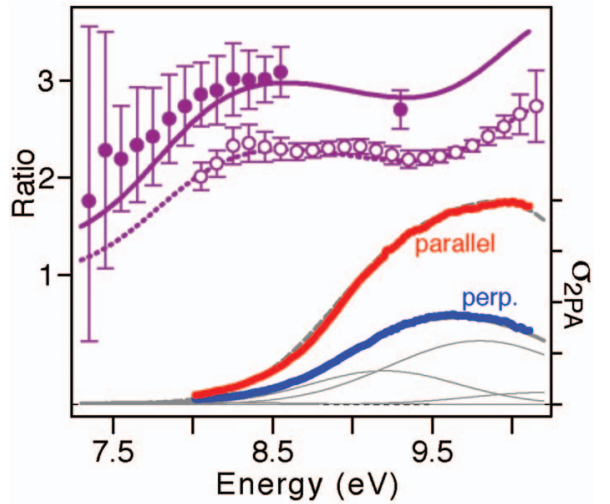

FIG. 7. (Color) Simulated polarization ratios and $2 \mathrm{PA}$ spectra from the second model in the text. Lines are the same as in Fig. 6(c).

6.2 versus $4.6 \mathrm{eV}$ pump photons. Although this is not a unique "fit" to the experimental ratios, the simulation nicely reproduces both the polarization ratio and the shapes of the parallel and perpendicular spectra.

An important difference from the simple model described in Sec. IV B 1 is the inclusion of a transition at 9.2 $\mathrm{eV}$. That transition is necessary to reproduce the relatively flat polarization ratio from about 8.3 to $9 \mathrm{eV}$, and also significantly improves the shape of the simulated 2PA spectra. In fact, the shape of the spectrum is a strong indication that there must be a transition between about 9 and $9.5 \mathrm{eV}$, even though there is not one in the calculation. The relatively large polarization ratio of the transition at $9.2 \mathrm{eV}$ indicates that it may be a totally symmetric $\left(A_{1}\right)$ transition if the $C_{2 v}$ point group remains valid in the liquid. In contrast, the $4 a_{1} \leftarrow 3 a_{1}{ }^{*}\left(\widetilde{B} 2^{1} A_{1}\right)$ transition at $9.8 \mathrm{eV}$ now has a somewhat smaller ratio than our gas-phase calculation suggests and, unlike the previous model, the low ratio for this state is responsible for the dip in the polarization ratio near $9.4 \mathrm{eV}$. The low ratio for the $\widetilde{B} 2^{1} A_{1}$ state may be a consequence of the splitting of the occupied $3 a_{1}$ orbitals in the liquid, ${ }^{55}$ or it may indicate the presence of a fifth, overlapping transition of low polarization ratio in the spectrum. It is not clear at this time how the splitting of the lower orbital in the liquid affects the polarization ratio, although calculations would be informative. The fourth and highest energy state at $10.2 \mathrm{eV}$ has a similar energy and intensity for both simulations, and is likely to have $A_{1}$ symmetry because of the very large ratio. Below, we consider how the liquid environment affects the ground and excited states of water and attempt to make a more definitive assignment of the previously unassigned transitions.

\section{Fate of gas-phase excited states in liquid water and tentative assignment of transitions}

The nature of the transition at $9.2 \mathrm{eV}$, and indeed the character of all of the transitions in the 2PA spectrum, depends on the fate of Rydberg excited states upon condensation. Little is known about those states in liquid water except that there are no sharp absorption bands such as the ones in the gas phase. ${ }^{57}$ Typically, the lowest Rydberg transition of an atom or a molecule in the condensed phase shifts to 
TABLE II. Parameters for the simulated polarization ratio in Fig. 7.

\begin{tabular}{lccccc}
\hline \hline Transition & $\begin{array}{c}\text { Energy } \\
(\mathrm{eV})\end{array}$ & $\begin{array}{c}\text { FWHM } \\
(\mathrm{eV})\end{array}$ & $\begin{array}{c}\sigma_{\text {para }} \\
(\mathrm{GM})\end{array}$ & $\begin{array}{c}\sigma_{\text {perp }} \\
(\mathrm{GM})\end{array}$ & $\left(\sigma_{\text {para }} / \sigma_{\text {perp }}\right)$ \\
\hline$\tilde{A} 1^{1} B_{1}$ & $8.3^{\mathrm{a}}$ & $1.0^{\mathrm{a}}$ & 0.01 & 0.01 & 0.9 \\
$\ldots$ & 9.2 & 1.1 & 1.8 & 0.7 & 2.7 \\
$\widetilde{B} 2^{1} A_{1}$ & $9.8^{\mathrm{b}}$ & 1.3 & 1.9 & 1.3 & 1.5 \\
$\ldots$ & 10.2 & 1.1 & 2.1 & 0.2 & 9.0 \\
\hline \hline
\end{tabular}

${ }^{\mathrm{a}}$ From the linear absorption spectrum (e.g., Ref. 24).

${ }^{\mathrm{b}}$ From the relative binding energies of the $1 b_{1}$ and $3 a_{1}{ }^{*}$ orbitals in the PE spectrum (Ref. 31). See text for details.

higher energy due to confinement of the upper (Rydberg) orbital by the solvent. This picture of a compressed Rydberg orbital is often used to describe the $0.9 \mathrm{eV}$ shift of the $\widetilde{A} 1^{1} B_{1}$ transition in the 1PA spectrum of water. ${ }^{30,34}$ Furthermore, the decreasing experimental 2PA polarization ratio at low energy [Fig. 5(b)] is consistent with the low ratio that we calculate for the $\widetilde{A} 1{ }^{1} B_{1}$ state of the isolated molecule, and supports the notion that the character of that state is not significantly perturbed in the liquid. Both $\widetilde{A} 1^{1} B_{1}$ and $\widetilde{B} 2^{1} A_{1}$ share the same $3 s / 4 a_{1}$ upper orbital, so it is likely that the $\widetilde{B} 2^{1} A_{1}$ state has a similar fate. An important difference is that there are two components for the $4 a_{1} \leftarrow 3 a_{1}\left(\widetilde{B} 2^{1} A_{1}\right)$ transition because of the electronic splitting of occupied $3 a_{1}$ orbitals in the condensed phase.

The identity and location of other transitions are more difficult to ascertain because it is unclear what happens to the higher unoccupied orbitals in liquid water. In contrast with the lowest member of a Rydberg series, the upper Rydberg states (and the vertical ionization potential) may shift to lower energy in the condensed phase due to polarization stabilization of the cation. ${ }^{58}$ Indeed, the first two ionization potentials of liquid water $\left(11.2 \mathrm{eV}\right.$ for $1 b_{1}$ and $13.5 \mathrm{eV}$ for $\left.3 a_{1}\right)$ are significantly lower than in the gas phase (12.6 and $14.8 \mathrm{eV}$, respectively). ${ }^{31}$ In other words, all other Rydberglike states that are correlated with the $\left(1 b_{1}\right)^{-1}$ hole would be compressed into a narrow energy range between the lowest state at $8.3 \mathrm{eV}$ and the vertical ionization potential at $11.2 \mathrm{eV}$. A shift to lower energy for all but the lowest Rydberg-like states would be a result of the diffuse character of the upper Rydberg orbitals, which are molecule centered, but encompass several neighboring solvent molecules and thus expose the cation. Even though the local solvent structure perturbs the instantaneous shape of the electronic wave function, the average electron density for many configurations reflects the Rydberg-like character $(s, p$, etc.). States correlated with the $\left(3 a_{1}\right)^{-1}$ cation should behave in a similar manner, but only the lowest energy transition at $9.8 \mathrm{eV}$ is likely to be in the range of our experiment. In any case, a large change in the equilibrium solvent structure between the ground and excited states will lead to significant spectral broadening of Rydberglike transitions in the condensed phase. ${ }^{29,58}$ The broadening increases and the transition strength decreases with increasing principle quantum number, so we do not expect to observe states with $n>3$.

The transition near $9.2 \mathrm{eV}$ therefore could originate as one of the gas-phase $\left(1 b_{1}\right)^{-1}$ Rydberg states that is broadened and shifted to very low energy in the liquid. Two possible candidates are the $2 b_{2} \leftarrow 1 b_{1}\left(1^{1} A_{2}\right)$ and $5 a_{1} \leftarrow 1 b_{1}\left(2^{1} B_{1}\right)$ transitions, although the polarization ratio of the $9.2 \mathrm{eV}$ state is larger than the upper limit of $4 / 3$ for nontotally symmetric states. The polarization ratio may not match the purely gasphase result because the spatially extended upper orbital experiences a local liquid environment that deviates from $C_{2 v}$ symmetry. The larger ratio at $9.2 \mathrm{eV}$ would reflect electronic mixing of the Rydberg states induced by the local asymmetry of the liquid, from which the $9.2 \mathrm{eV}$ transition obtains some of the character of the strongly polarized $2 b_{1} \leftarrow 1 b_{1}\left(3^{1} A_{1}\right)$ transition. The very large polarization ratio of the transition at $10.2 \mathrm{eV}$ points to the gas-phase $2 b_{1} \leftarrow 1 b_{1}\left(3^{1} A_{1}\right)$ Rydberg excitation as the primary origin of that state. A number of alcohols, alkanes and chlorosubstituted alkanes also have relatively large 2PA polarization ratios $(>2.5)$ for electronic bands that derive from nontotally symmetric states in the isolated molecule, and it is possible that symmetry breaking commonly leads to larger polarization ratios in the liquid than in the gas phase. ${ }^{59}$

A complete Rydberg-like picture of water would include transitions to all of the oxygen $3 p$ upper orbitals $\left(3 p_{y} / 2 b_{2}\right.$, $3 p_{z} / 5 a_{1}$, and $\left.3 p_{x} / 2 b_{1}\right)$, as well as the two transitions to $3 s / 4 a_{1}$ that were already assigned (see Fig. 2). Including all of those states requires a five-state model rather than the four states that we use above. As mentioned earlier, the surprisingly low polarization ratio for the $\widetilde{B} 2^{1} A_{1}$ state at $9.8 \mathrm{eV}$ in the second simulation could be the result of accidental overlap of that transition with the missing fifth Rydberg-like transition. Unlike the transition at $9.2 \mathrm{eV}$, the fifth transition at $9.8 \mathrm{eV}$ would require a low polarization ratio. The $3 p_{y} / 2 b_{2}$ orbital has some valence antibonding character, which, being more localized, potentially reduces the extent of mixing with the purely $3 p$ Rydberg-like excited states, making the $2 b_{2} \leftarrow 1 b_{1}\left(1^{1} A_{2}\right)$ transition a leading candidate for the possible fifth transition. By elimination, that assignment would suggest that the transition at $9.2 \mathrm{eV}$ mainly comes from the $5 a_{1} \leftarrow 1 b_{1}\left(2^{1} B_{1}\right)$ Rydberg transition with some mixing with the $2 b_{1} \leftarrow 1 b_{1}\left(3^{1} A_{1}\right)$ transition. This assignment is consistent with weak 1PA in this energy region $[9.2 \mathrm{eV}$ is near the minimum of the 1PA absorption band in Fig. 1(b)] because the one-photon oscillator strength of the $2^{1} B_{1}$ transition is nearly an order of magnitude lower than the other onephoton allowed transitions in Table I. Thus, the Rydberg-like picture of liquid water includes transitions to the (mixed) 
oxygen $3 p$ orbitals centered at $9.2,9.8$, and $10.2 \mathrm{eV}$, as well as the transitions to $3 s / 4 a_{1}$ at 8.3 and $9.8 \mathrm{eV}$.

The characteristics of the Rydberg-like transition at 10.2 $\mathrm{eV}$ are partly a result of the delocalized excited state, with the large polarization ratio originating from a spherically symmetric atomic oxygen picture. As we mentioned above, the spherically symmetric limit gives an infinite polarization ratio for transitions that preserve the symmetry of the wave function (i.e., $p_{x}-p_{x}$ ). The spherically symmetric atomic oxygen basis set may still be appropriate for highly delocalized orbitals in the disordered liquid, justifying the Rydberglike picture. Such a picture is similar to one describing the excited states of water as Wannier excitons. ${ }^{26,29}$ Borrowed from the solid-state literature, Wannier excitons are characterized by a delocalized electron that is weakly bound to a positively charged hole. ${ }^{60}$ The excitons lie in a series converging on the conduction band, with the solvent partially screening the electron from the hole. The lifetime of the exciton is determined by the mean free path of the electron in the liquid ${ }^{61}$ and scattering of the electron would cause significant lifetime broadening of the transition. Similar to the picture of Rydberg excited states, Wannier exciton states would appear as broad transitions between the energy of the lowest excited state $(8.3 \mathrm{eV})$ and the bottom of the conduction band near $11 \mathrm{eV}$. In both pictures, the optical transition promotes an electron from a localized molecular orbital to a highly delocalized upper orbital.

The behavior of the 2PA spectrum above $10.2 \mathrm{eV}$ is beyond the range of our measurement, but the perpendicularly polarized spectrum of Thomsen et $_{\text {al. }}{ }^{28}$ indicates that the 2PA cross section increases again after reaching a shallow minimum around $10.4 \mathrm{eV}$. Their spectrum extends to about $10.8 \mathrm{eV}$, which is also the energy of our single-point measurement of the polarization ratio. At that energy we obtain a polarization ratio of only $2.4( \pm 0.2)$, well below the expected value if the $3^{1} A_{1}$-like state were to dominate that region of the spectrum. The experimental ratio is in reasonable agreement with the calculated ratio for the $4 a_{1} \leftarrow 3 a_{1}$ $\left(\widetilde{B} 2^{1} A_{1}\right)$ transition in the isolated molecule, and the excitation energy is relatively close to the location of the upper component of that state. An excitation energy of $10.8 \mathrm{eV}$ is also very close to the vertical ionization threshold $(\sim 11 \mathrm{eV})$, therefore 2PA at that energy may include a contribution from excitation directly into the conduction band. ${ }^{15}$

For strongly interacting molecules in a liquid, collective excitation and charge transfer among adjacent species may play an increasing role at higher energy. ${ }^{26}$ In this picture there are excited states that bear little resemblance to sitecentered Rydberg states of the isolated molecule, and instead the excited states are composed of unoccupied molecular orbitals from more than one water molecule. Two recent, independent measurements of the spectator Auger-electron spectrum of liquid water show experimentally that the electron becomes increasingly delocalized as the excitation energy increases. ${ }^{33,62}$ Even for the lowest excited state of condensed water, Hahn et al..$^{34}$ showed that the excited electron is somewhat delocalized onto neighboring molecules, although the excited state is still located primarily on a single water molecule.

\section{Connecting 2PA and XA spectroscopy}

XA spectroscopy provides an important point of comparison with the optical spectrum because the $\mathrm{x}$-ray measurements probe the empty orbitals of water via transitions from the compact, localized $1 a_{1}$ (oxygen $1 s$ ) orbital. ${ }^{63}$ The XA spectrum of the liquid is usually described in terms of preedge, main-edge, and postedge transitions at 535.0, 537.6, and $540.5 \mathrm{eV}$, respectively, with an additional contribution from the direct excitation of an electron into the conduction band above $538.1 \mathrm{eV}^{62}$ Although the interpretation of the pre-edge transition with respect to the hydrogen-bonding structure of liquid water is debatable,${ }^{64-66}$ it is well established that the absorption band arises from a $4 a_{1} \leftarrow 1 a_{1}$ transition, and therefore is analogous to the lowest energy $4 a_{1} \leftarrow 1 b_{1}\left(\tilde{A} 1^{1} B_{1}\right)$ transition in the optical spectrum.

According to the results from our second model of the 2PA spectrum, the two-photon $4 a_{1} \leftarrow 1 b_{1}\left(\widetilde{A} 1^{1} B_{1}\right)$ transition is even weaker in the liquid than the gas-phase calculations suggest. The simulated cross section is fully two orders of magnitude smaller than for the higher energy transitions in Table II. A very weak transition in the condensed phase is in line with the XA spectrum of ice, where the $4 a_{1} \leftarrow 1 a_{1}$ transition near $535 \mathrm{eV}$ is virtually nonexistent. ${ }^{63-65}$ There, it was argued that the fully coordinated, tetrahedral hydrogenbonding geometry eliminates any residual oxygen $3 p$ character from the $4 a_{1}$ upper orbital, thus making the $4 a_{1} \leftarrow 1 s$ $\mathrm{x}$-ray transition unfavorable. Because the two-photon transition from $1 b_{1}$ is also sensitive to the amount of oxygen $3 p$ character in the upper orbital, the $4 a_{1} \leftarrow 1 b_{1}\left(\widetilde{A} 1^{1} B_{1}\right)$ cross section would also be reduced in the condensed phase. Asymmetric rehybridization due to broken hydrogen bonds in the disordered liquid ${ }^{63-66}$ should therefore have a similar effect in both spectra, giving slightly stronger transitions compared with bulk ice, but weaker than the isolated molecule. Temperature-dependent measurements and a comparison with the 2PA spectrum of ice would facilitate a more thorough analysis of the role of hydrogen bonding in the optical spectroscopy of water.

The assignment of the XA main-edge and postedge is less clear than for the pre-edge transition because of the relatively featureless spectrum and higher density of states. Based on the gas-phase XA spectrum, the main edge sometimes is described as the $2 b_{2} \leftarrow 1 a_{1}$ excitation and the postedge as a transition to higher Rydberg-like orbitals. ${ }^{62}$ If the main edge of the XA spectrum at $537.6 \mathrm{eV}$ does indeed reflect the location of the $2 b_{2}$ orbital relative to the $4 a_{1}$ preedge feature, then the corresponding $2 b_{2} \leftarrow 1 b_{1}\left(1^{1} A_{2}\right)$ transition could be located as much as $2.6 \mathrm{eV}$ above the $4 a_{1} \leftarrow 1 b_{1}\left(\widetilde{A} 1^{1} B_{1}\right)$ transition. That would place $2 b_{2} \leftarrow 1 b_{1}$ $\left(1^{1} A_{2}\right)$ near $10.9 \mathrm{eV},{ }^{67}$ which is outside the window of our experiment. Thus, it is possible that the $2 b_{2} \leftarrow 1 b_{1}\left(1^{1} A_{2}\right)$ transition is at higher energy than we can observe in our experimental spectrum. In contrast, the condensed phase calculations predict that $2 b_{2}$ lies only $1.65 \mathrm{eV}$ above $3 s / 4 a_{1}$ (see Table I), ${ }^{39}$ placing the transition slightly below $10 \mathrm{eV}$. The lower energy assignment is in excellent agreement with the Rydberg-like picture of excited states that includes a fifth transition near $9.8 \mathrm{eV}$. In either case it seems unlikely that 
the transition at $9.2 \mathrm{eV}$ is the one-photon-forbidden, twophoton-allowed $2 b_{2} \leftarrow 1 b_{1}\left(1^{1} A_{2}\right)$ transition because of its low energy.

Considering the new information from the experimental 2PA polarization ratio, it is unlikely that the "main edge" of the XA spectrum is in fact composed of a single X-ray transition. All of the Rydberg-like oxygen $3 p$ orbitals should contribute to the XA spectrum. Therefore, in addition to the $4 a_{1} \leftarrow 1 a_{1}$ excitation in the pre-edge region, the four (five)state 2PA model indicates that there are two (three) other $\mathrm{x}$-ray transitions contributing to the rising edge of the mainedge absorption band. These transitions may have different relative intensities than in the $2 \mathrm{PA}$ spectrum due to the different overlap of $1 a_{1}$ and $1 b_{1}$ with the upper orbitals. Importantly, there is no analog of the $4 a_{1} \leftarrow 3 a_{1}\left(\widetilde{B} 2^{1} A_{1}\right)$ transition in the XA spectrum because the electron comes from a different lower orbital than the other optical transitions, ${ }^{62}$ as illustrated in Fig. 2. In other words, four of the five states in the Rydberg-like 2PA model would play a role in the low energy region of the XA spectrum that includes the pre-edge and part of the main edge. Extending our analysis to higher energies, it seems likely that the postedge region of the XA spectrum also includes multiple transitions, but those higher energy transitions are beyond the scope of our 2PA measurements.

\section{E. Connecting 2PA spectroscopy with the ionization dynamics}

The character of the excited state is intimately connected with the ionization and dissociation dynamics of liquid water. The ionization and dissociation yields, and even the mechanisms, change as a function of the excitation energy. ${ }^{13-15,17,68-70}$ Interestingly, the ionization products have been observed for excitation energies as low as $6-6.5 \mathrm{eV},{ }^{16,17}$ even though vertical ionization is only possible above $\sim 11 \mathrm{eV} .^{71}$ For two-photon excitation, the ionization yield increases rapidly above $\sim 8.5 \mathrm{eV},{ }^{17,72}$ but the electron ejection length, which reflects the mechanism for the ionization, starts rising only above $9.5 \mathrm{eV}^{14,15}$ (The ejection length is the distance that the electron travels before it is trapped and solvated by the liquid.) The resulting picture for the ionization mechanism is one in which nuclear motion plays a central role at low excitation energies, with the conduction band becoming increasingly important at higher energies. ${ }^{13-15} \mathrm{Im}$ portantly, however, many of these experimental inputs use two-photon excitation and the current work shows that the mode of excitation alters the initially accessed electronic state.

At low energies (below about $10 \mathrm{eV}$ ) we can distinguish essentially two types of states for liquid water, those that involve promotion to the $3 s / 4 a_{1}$ orbital, and those going to $3 p$ Rydberg-like orbitals. We have argued that the $\tilde{A} 1^{1} B_{1}$ and $\widetilde{B} 2^{1} A_{1}$ states remain relatively compact, retaining much of the $3 s / 4 a_{1}$ character from the isolated molecule limit. Both of these states are directly dissociative in the gas phase, whereas the $3 p$ Rydberg states are not. In comparison, the $3 p \leftarrow 1 b_{1}$ states are more spatially extended and become mixed due to the solvent asymmetry. However, the highest energy state in our simulation (at $10.2 \mathrm{eV}$ ), still retains a very high polarization ratio reflecting the site-centered $p_{x}-p_{x}$ atomic parentage. As the energy of the upper orbital approaches the energy of the vertical conduction band it will have increasing conduction band character. Within the Rydberg picture, this means that the $3 p \leftarrow 1 b_{1}$ manifold of states just below the $\left(1 b_{1}\right)^{-1}$ vertical conduction band mixes with that continuum. The increasing conduction band character leads to more efficient ionization and a larger electron ejection length.

For excitation with two photons, transitions from $1 b_{1}$ to the spatially extended Rydberg-derived excited states with $3 p$ character are more prominent than the transition ending in the $3 s / 4 a_{1}$ upper orbital. In other words, two-photon excitation can access the $2^{1} B_{1}$ and $1^{1} A_{2}$ Rydberg-like states (in addition to $\widetilde{B} 2^{1} A_{1}$ ), whereas both of these Rydberg transitions are weak in 1PA. Thus, the 2PA spectrum rises more rapidly above $9 \mathrm{eV}$ than the 1PA spectrum. We expect the $3 p$ character to enhance the likelihood of two-photon ionization and, indeed, the two-photon induced ionization yield rises strongly in this energy region. In comparison, the $\widetilde{B} 2^{1} A_{1}$ excited state is also accessible and it is dissociative in the gas phase. We recently studied the dissociation dynamics following two-photon excitation at $8.3 \mathrm{eV}$, where dissociation is at least seven times more likely than ionization. ${ }^{70}$ Although in that work we described the dissociation dynamics primarily in terms of the $\tilde{A} 1^{1} B_{1}$ potential energy surface, it is now clear that because the two-photon cross section for that state is weak, other states must play a role even for $8.3 \mathrm{eV}$ excitation, including the state at $9.2 \mathrm{eV}$ and the low energy edge of the $\widetilde{B} 2^{1} A_{1}$ state. Unlike the $\widetilde{B} 2^{1} A_{1}$ state, the $9.2 \mathrm{eV}$ state is not directly dissociative and is likely coupled to the conduction band by virtue of its more diffuse $3 p$ character. On the other hand, the $\widetilde{B} 2^{1} A_{1}$ state preferentially dissociates because it is very weakly coupled to the $\left(3 a_{1}\right)^{-1}$ conduction band due to the large energy difference. The $\widetilde{B} 2^{1} A_{1}$ state is even more unlikely to access the $\left(1 b_{1}\right)^{-1}$ conduction band because that would require a two-electron process. The underlying $\widetilde{B} 2^{1} A_{1}$ state, which has components at 9.8 and 11.4 $\mathrm{eV}$, could explain the persistence of the dissociation channel for energies as high as $\sim 11 \mathrm{eV} .^{72}$ The upper component of $\widetilde{B} 2^{1} A_{1}$ however will be more strongly coupled to the $\left(3 a_{1}\right)^{-1}$ continuum than the lower component.

Considering all of these points, and recalling that all two-photon ionization yield and ejection length measurements to date use a single, linearly polarized laser beam for excitation, some important observations emerge. Comparing the ionization and dissociation yields as a function of the relative polarization of the two photons being absorbed would potentially clarify the relative contribution of each state. States with smaller polarization ratios are less prominent for parallel two-photon excitation, instead favoring the transitions with larger polarization ratios. Likewise, our analysis suggests that ionization and dissociation yields should be different at the same excitation energy for one- 
photon versus two-photon excitation. Similarly, the pathway for ionization should differ, as reflected in the ejection length.

\section{CONCLUSION}

2PA spectroscopy provides new insight about the electronic structure of liquid water that is not available from the one-photon spectrum. In particular, the polarization dependence of the 2PA cross section reveals the locations and, to some extent, the identities of the electronically excited states. The energy dependence of the polarization ratio suggests that at least four states contribute to the 2PA spectrum in the energy range of our experiment. Two of the states have the same $3 s / 4 a_{1}$ upper orbital and are assigned as $\widetilde{A} 1{ }^{1} B_{1}$ and $\widetilde{B} 2^{1} A_{1}$ based on previous measurements of the liquid 1PA and PE spectra. The two-photon $4 a_{1} \leftarrow 1 b_{1}\left(\widetilde{A} 1^{1} B_{1}\right)$ transition is very weak because of the atomic oxygen character of that excitation. The small 2PA cross section and concurrent shift to higher energy relative to the isolated molecule has important practical consequences, as it effectively gives water a wider window of transparency in two-photon versus one-photon experiments. The wider two-photon "band gap" of liquid water makes it possible to study the ultrafast dynamics of aqueous solutes without a significant contribution from either two-photon excitation (and thus ionization) of the solvent or an overwhelming "coherent artifact" at short times originating from pump+probe $2 \mathrm{PA}$ of the solvent, even for intense femtosecond pump pulses reaching into the near UV.

The electronic structure of water is more complicated at higher energy, where there are several overlapping 2PA transitions. Using gas-phase calculations as a well-defined reference point, we find that a Rydberg-like picture of the electronically excited states provides a good description of the experimental spectrum, including the energy dependence of the polarization ratio. In that picture, the mixed Rydberg-like states are coupled to the conduction band, helping to explain the rapidly increasing ionization yield above $\sim 8.5 \mathrm{eV}$. A more detailed picture of electronically excited liquid water requires a thorough theoretical treatment of the electronic wave function in the excited states that is well beyond the scope of this work. At the very least, new details obtained from the 2PA spectrum provide an important benchmark for those calculations. We stress that any complete description of the electronic structure of liquid water must include a comprehensive treatment of one- and two-photon absorption, $\mathrm{XA}$, and PE spectroscopies.

Our results demonstrate the power of broadband 2PA measurements for unraveling the electronic spectroscopy of complex systems. The different selection rules compared to 1PA spectroscopy and the polarization dependence of the 2PA spectrum open a new window on the excited states, which is an invaluable tool for resolving relatively featureless spectra. This technique will be particularly useful in the one-photon vacuum-UV range, where the spectrum typically involves several overlapping transitions.

\section{ACKNOWLEDGMENTS}

This work was supported by the National Science Foundation (No. CHE-0617060). We thank Anna Krylov for helpful discussions regarding calculation of two-photon properties, and for computational resources through the Center for Computational Studies of Electronic Structure and Spectroscopy of Open-Shell and Electronically Excited Species (iopenshell.usc.edu) supported by the NSF CRIF:CRF program (Nos. CHE-0625419, CHE-0624602, and CHE-062537).

${ }^{1}$ P. R. Monson and W. M. McClain, J. Chem. Phys. 53, 29 (1970)

${ }^{2}$ W. M. McClain, J. Chem. Phys. 55, 2789 (1971).

${ }^{3}$ W. M. McClain, Acc. Chem. Res. 7, 129 (1974).

${ }^{4}$ M. J. Wirth, A. Koskelo, and M. J. Sanders, Appl. Spectrosc. 35, 14 (1981).

${ }^{5}$ M. Albota, D. Beljonne, J. L. Bredas, J. E. Ehrlich, J. Y. Fu, A. A. Heikal, S. E. Hess, T. Kogej, M. D. Levin, S. R. Marder, D. McCord-Maughon, J. W. Perry, H. Rockel, M. Rumi, C. Subramaniam, W. W. Webb, X. L. $\mathrm{Wu}$, and C. $\mathrm{Xu}$, Science 281, 1653 (1998).

${ }^{6}$ G. S. He, T. C. Lin, and P. N. Prasad, Opt. Express 10, 566 (2002).

${ }^{7}$ M. Balu, J. Hales, D. J. Hagan, and E. W. Van Stryland, Opt. Express 12, 3820 (2004)

${ }^{8}$ L. De Boni, A. A. Andrade, L. Misoguti, C. R. Mendonca, and S. C. Zilio, Opt. Express 12, 3921 (2004).

${ }^{9}$ R. A. Negres, J. M. Hales, D. J. Hagan, and E. W. Van Stryland, IEEE J. Quantum Electron. 38, 1205 (2002).

${ }^{10}$ R. A. Negres, J. M. Hales, A. Kobyakov, D. J. Hagan, and E. W. Van Stryland, Opt. Lett. 27, 270 (2002).

${ }^{11}$ S. Yamaguchi and T. Tahara, Chem. Phys. Lett. 376, 237 (2003).

${ }^{12}$ S. Yamaguchi and T. Tahara, Chem. Phys. Lett. 390, 136 (2004).

${ }^{13}$ M. U. Sander, K. Luther, and J. Troe, J. Phys. Chem. 97, 11489 (1993).

${ }^{14}$ R. A. Crowell and D. M. Bartels, J. Phys. Chem. 100, 17940 (1996).

${ }^{15}$ C. G. Elles, A. E. Jailaubekov, R. A. Crowell, and S. E. Bradforth, J. Chem. Phys. 125, 044515 (2006).

${ }^{16}$ J. W. Boyle, J. A. Ghormley, C. J. Hochanadel, and J. F. Riley, J. Phys. Chem. 73, 2886 (1969).

${ }^{17}$ D. M. Bartels and R. A. Crowell, J. Phys. Chem. A 104, 3349 (2000) (and references therein).

${ }^{18}$ R. Mota, R. Parafita, A. Giuliani, M. J. Hubin-Franskin, J. M. C. Lourenco, G. Garcia, S. V. Hoffmann, N. J. Mason, P. A. Ribeiro, M. Raposo, and P. Limao-Vieira, Chem. Phys. Lett. 416, 152 (2005).

${ }^{19}$ R. S. Mulliken, Chem. Phys. Lett. 46, 197 (1977).

${ }^{20}$ P. S. Bagus, C. Woll, and E. S. Ilton, Chem. Phys. Lett. 428, 207 (2006).

${ }^{21}$ M. Rubio, L. Serrano-Andres, and M. Merchan, J. Chem. Phys. 128, 104305 (2008).

${ }^{22}$ G. H. F. Diercksen, W. P. Kraemer, T. N. Rescigno, C. F. Bender, B. V. McKoy, S. R. Langhoff, and P. W. Langhoff, J. Chem. Phys. 76, 1043 (1982).

${ }^{23}$ H. Hayashi, N. Watanabe, Y. Udagawa, and C. C. Kao, Proc. Natl. Acad. Sci. U.S.A. 97, 6264 (2000).

${ }^{24}$ J. M. Heller, Jr., R. N. Hamm, R. D. Birkhoff, and L. R. Painter, J. Chem. Phys. 60, 3483 (1974).

${ }^{25}$ G. D. Kerr, M. W. Williams, R. D. Birkhoff, R. N. Hamm, and L. R. Painter, Phys. Rev. A 5, 2523 (1972).

${ }^{26}$ K. Kobayashi, J. Phys. Chem. 87, 4317 (1983).

${ }^{27}$ P. Han and D. M. Bartels, J. Phys. Chem. 94, 5824 (1990).

${ }^{28}$ C. L. Thomsen, D. Madsen, S. R. Keiding, J. Thogersen, and O. Christiansen, J. Chem. Phys. 110, 3453 (1999).

${ }^{29}$ M. B. Robin, Higher Excited States of Polyatomic Molecules (Academic, New York, 1974).

${ }^{30}$ M. Chergui and N. Schwentner, Chem. Phys. Lett. 219, 237 (1994).

${ }^{31}$ B. Winter, R. Weber, W. Widdra, M. Dittmar, M. Faubel, and I. V. Hertel, J. Phys. Chem. A 108, 2625 (2004).

${ }^{32}$ E. Keszei and J. P. Jay-Gerin, Can. J. Chem. 70, 21 (1992).

${ }^{33}$ D. Nordlund, H. Ogasawara, H. Bluhm, O. Takahashi, M. Odelius, M. Nagasono, L. G. M. Pettersson, and A. Nilsson, Phys. Rev. Lett. 99, 217406 (2007).

${ }^{34}$ P. H. Hahn, W. G. Schmidt, K. Seino, M. Preuss, F. Bechstedt, and J. Bernholc, Phys. Rev. Lett. 94, 037404 (2005).

${ }^{35}$ V. Garbuio, M. Cascella, L. Reining, R. Del Sole, and O. Pulci, Phys. Rev. Lett. 97, 137402 (2006). 
${ }^{36}$ A. Hermann, W. G. Schmidt, and P. Schwerdtfeger, Phys. Rev. Lett. 100, 207403 (2008).

${ }^{37}$ K. Laasonen, M. Sprik, M. Parrinello, and R. Car, J. Chem. Phys. 99, 9080 (1993).

${ }^{38}$ O. Christiansen, T. M. Nymand, and K. V. Mikkelsen, J. Chem. Phys. 113, 8101 (2000).

${ }^{39}$ M. J. Paterson, J. Kongsted, O. Christiansen, K. V. Mikkelsen, and C. B. Nielsen, J. Chem. Phys. 125, 184501 (2006).

${ }^{40}$ B. D. Bursulaya, J. Jeon, C. N. Yang, and H. J. Kim, J. Phys. Chem. A 104, 45 (2000).

${ }^{41}$ P. Cabral do Couto, S. G. Estacio, and B. J. C. Cabral, J. Chem. Phys. 123, 054510 (2005)

${ }^{42}$ D. N. Nikogosyan, A. A. Oraevsky, and V. I. Rupasov, Chem. Phys. 77, 131 (1983).

${ }^{43}$ A. Reuther, A. Laubereau, and D. N. Nikogosyan, J. Phys. Chem. 100, 16794 (1996).

${ }^{44}$ A. Reuther, A. Laubereau, and D. N. Nikogosyan, Opt. Commun. 141, 180 (1997).

${ }^{45}$ S. Pommeret, F. Gobert, A. Mostafavi, I. Lampre, and J. C. Mialocq, J. Phys. Chem. A 105, 11400 (2001).

${ }^{46}$ A. Dragomir, J. G. McInerney, D. N. Nikogosyan, and A. A. Ruth, IEEE J. Quantum Electron. 38, 31 (2002).

${ }^{47}$ M. J. Tauber, R. A. Mathies, X. Y. Chen, and S. E. Bradforth, Rev. Sci. Instrum. 74, 4958 (2003).

${ }^{48}$ S. A. Kovalenko, A. L. Dobryakov, J. Ruthmann, and N. P. Ernsting, Phys. Rev. A 59, 2369 (1999).

${ }^{49}$ DALTON, a molecular electronic structure program, Release 2.0, 2005, see http://www.kjemi.uio.no/software/dalton/dalton.html.

${ }^{50}$ G. Herzberg, Molecular Spectra and Molecular Structure: II. Infrared and Raman Spectra of Polyatomic Molecules (Krieger, Malabar, 1991), p. 489.

${ }^{51}$ C. B. Nielsen, S. Rettrup, and S. P. A. Sauer, J. Chem. Phys. 124, 114108 (2006).

${ }^{52}$ We use a different excitation energy, which will have a subtle effect on the shape of the 2PA spectrum due to preresonant enhancement.

${ }^{53}$ The slightly lower polarization purity of the $6.2 \mathrm{eV}$ beam does not account for the substantially lower polarization ratio compared to $4.6 \mathrm{eV}$ excitation.
${ }^{54}$ P. Hunt, M. Sprik, and R. Vuilleumier, Chem. Phys. Lett. 376, 68 (2003).

${ }^{55}$ D. Nordlund, M. Odelius, H. Bluhm, H. Ogasawara, L. G. M. Pettersson, and A. Nilsson, Chem. Phys. Lett. 460, 86 (2008).

${ }^{56}$ G. P. Parravicini and L. Resca, Phys. Rev. B 8, 3009 (1973).

${ }^{57}$ A. Bernas, C. Ferradini, and J. P. Jay-Gerin, Chem. Phys. 222, 151 (1997).

${ }^{58}$ F. Vigliotti and M. Chergui, Eur. Phys. J. D 10, 379 (2000).

${ }^{59}$ Y. Zhang, C. G. Elles, C. A. Rivera, and S. E. Bradforth (unpublished).

${ }^{60}$ R. S. Knox, Theory of Excitons (Academic, New York, 1963).

${ }^{61}$ S. A. Rice and J. Jortner, J. Chem. Phys. 44, 4470 (1966).

${ }^{62}$ B. Winter, U. Hergenhahn, M. Faubel, O. Bjorneholm, and I. V. Hertel, J. Chem. Phys. 127, 094501 (2007).

${ }^{63}$ M. Cavalleri, H. Ogasawara, L. G. M. Pettersson, and A. Nilsson, Chem. Phys. Lett. 364, 363 (2002).

${ }^{64}$ S. Myneni, Y. Luo, L. A. Naslund, M. Cavalleri, L. Ojamae, H. Ogasawara, A. Pelmenschikov, P. Wernet, P. Vaterlein, C. Heske, Z. Hussain, L. G. M. Pettersson, and A. Nilsson, J. Phys.: Condens. Matter 14, L213 (2002).

${ }^{65}$ P. Wernet, D. Nordlund, U. Bergmann, M. Cavalleri, M. Odelius, H. Ogasawara, L. A. Naslund, T. K. Hirsch, L. Ojamae, P. Glatzel, L. G. M. Pettersson, and A. Nilsson, Science 304, 995 (2004).

${ }^{66}$ J. D. Smith, C. D. Cappa, K. R. Wilson, B. M. Messer, R. C. Cohen, and R. J. Saykally, Science 306, 851 (2004).

${ }^{67}$ The $2 b_{2}-1 b_{1}$ transition energy is mistakenly reported in Ref. 62 as 10.4 $\mathrm{eV}$, rather than $10.7 \mathrm{eV}$. Correcting for the different $4 a_{1}-1 b_{1}$ transition energies in the XA work and in the 1PA spectrum (8.1 vs $8.3 \mathrm{eV}$ ) gives a slightly higher value of $10.9 \mathrm{eV}$.

${ }^{68}$ M. U. Sander, K. Luther, and J. Troe, Ber. Bunsenges. Phys. Chem 97, 953 (1993).

${ }^{69}$ M. U. Sander, M. S. Gudiksen, K. Luther, and J. Troe, Chem. Phys. 258, 257 (2000).

${ }^{70}$ C. G. Elles, I. A. Shkrob, R. A. Crowell, and S. E. Bradforth, J. Chem. Phys. 126, 164503 (2007).

${ }^{71}$ Vertical ionization is defined here as the direct excitation of an electron into the conduction band of the liquid.

${ }^{72}$ A. Mozumder, Phys. Chem. Chem. Phys. 4, 1451 (2002). 